\title{
An imported case of e-cigarette or vaping associated lung injury in Barcelona
}

\section{To the Editor:}

Very recently, LAYDEN et al. [1] published the first series of 53 patients with acute lung injury associated with the use of e-cigarettes (also known as vaping) in Wisconsin and Illinois, USA. To our knowledge, only a few cases of e-cigarette, or vaping, associated lung injury (EVALI) have been reported outside the USA (in Canada and Japan) and, so far, none in Europe [2]. Here we present the case of a 31-year-old woman who lived in Chicago (Illinois, USA) and arrived in Barcelona (Spain) on vacation on September 17, 2019 and, as detailed below, suffered EVALI. To our knowledge, this is the first case of EVALI reported in Europe and, given the current trend towards globalisation that allows individuals to travel to other countries easily, it highlights the need of being aware of the possibility of encountering, thus diagnosing and treating, a case of vaping-induced acute lung injury outside the USA.

The past medical history of the patient was unremarkable. She had been vaping nicotine daily for the last 3 months but, during the past week, she used nicotine salts using the same device. She attended the emergency room of Hospital Clinic in Barcelona, Spain after a 72-h history of fever, myalgia, dry cough, dyspnoea and fatigue. On admission, temperature was $38.5^{\circ} \mathrm{C}$, respiratory rate 26 breaths per min, heart rate 110 beats per min, and left crackles were audible on thoracic auscultation. Chest radiography showed left lower alveolar infiltrates. Arterial blood gases breathing $24 \% \mathrm{O}_{2}$ showed $P_{\mathrm{aO}_{2}} 44 \mathrm{mmHg}, P_{\mathrm{aCO}_{2}} 34 \mathrm{mmHg}$ and pH 7.33 . Leukocyte count was $11.8 \times 10^{9}$ per L ( $88 \%$ polymorphonuclear cells) and C-reactive protein levels $17 \mathrm{mg} \cdot \mathrm{dL}^{-1}$ (normal $<1 \mathrm{mg} \cdot \mathrm{dL}^{-1}$ ). Kidney and liver function were normal, and urine antigen testing for Streptococcus and Legionella, blood culture and HIV serology were all negative. Following an initial diagnose of community acquired pneumonia, treatment with ceftriaxone (2 g daily), azithromycin (500 mg daily) and methylprednisolone ( $40 \mathrm{mg}$ daily) was initiated. Yet, $72 \mathrm{~h}$ later, her clinical condition had deteriorated, and radiological chest infiltrates had worsened. A computed tomography (CT) chest scan (figure 1) showed bilateral lower lobe consolidation with air bronchograms, pleural effusion and peri-bronchial ground glass opacities in the upper and medium right lung. Pleural fluid characteristics were protein levels $17 \mathrm{~g} \cdot \mathrm{L}^{-1}, \mathrm{pH}$ 7.86, glucose $160 \mathrm{mg} \cdot \mathrm{dL}^{-1}$, lactate dehydrogenase $219 \mathrm{UI} \cdot \mathrm{L}^{-1}$, and neutrophil predominance (60\%); bacterial culture was negative. Bronchoalveolar lavage fluid (BALF) showed lipid laden macrophages (55\%), lymphocytes (28\%) and neutrophils (17\%). BALF culture and PCR assay for bacterial, fungal, and viral pathogens was negative. Considering the possibility of EVALI, methylprednisolone doses were increased to $40 \mathrm{mg}$ every $12 \mathrm{~h}$. During the next 7 days, her clinical condition improved progressively with complete resolution of radiographic opacities. 12 days after hospitalisation, the patient could be discharged home without supplemental oxygen and progressive tapering of oral prednisone. A few days later, the patient flew back to Chicago, USA, uneventfully.

This clinical case fulfils all the diagnostic criteria proposed by the Centers for Disease Control and Prevention (CDC) for EVALI [1], namely: 1) use of an e-cigarette (vaping) in 90 days before symptom onset; 2) pulmonary infiltrates (e.g. ground-glass opacities) on chest CT (figure 1) [3]; and 3) absence of pulmonary infection (i.e. negative respiratory viral panel, urine antigen testing for Streptococcus pneumoniae and Legionella, sputum, BALF or blood culture, and absence of HIV presence) or alternative plausible diagnoses (e.g. cardiac, rheumatologic or neoplastic process). The presence of lipid laden macrophages in BALF [4] is likely a marker of vaping but not a criteria for the diagnosis of EVALI. The clinical presentation of this patient is similar to those reported previously, albeit pleural effusion is unusual [1].

To our knowledge, this is the first case of EVALI reported in Europe. This contrasts with the recent epidemic seen in the USA [1]. As of 22 October, 2019, 1604 cases of EVALI have been reported to CDC, and 34 deaths have been confirmed. E-cigarettes are also widely used in Europe, both for leisure and as a smoking cessation 

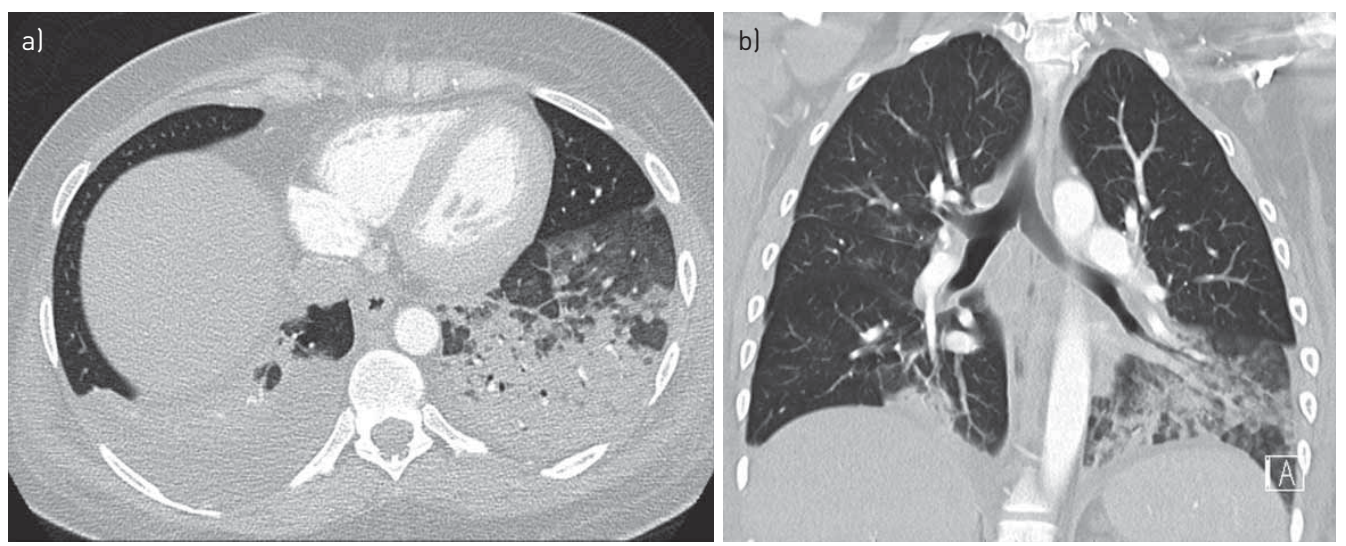

FIGURE 1 Thoracic computed tomography with i.v. contrast. a) Axial image and b) coronal reformation showing small right pleural effusion and bilateral foci of ground glass and consolidation with peri-bronchovascular, peri-lobular and lobar distribution.

strategy. For instance, e-cigarettes have now become the most common quitting aid for smokers in England [5], yet EVALI cases have not been reported in Europe to date. Reasons for this geographical discrepancy are unclear but may relate to the fact that many, but not all, of reported cases have been associated with vaping of cannabinoid-containing products and not simply nicotine $[1,6]$. Yet, due to the wide diversity and unregulated nature of vaping devices, products and practice habits among users, the specific chemical compounds directly causing lung damage remain obscure in many cases [6]. In fact, the patient reported here vaped "freebase" nicotine daily for the previous 3 months, which is the nicotine form ordinarily used in e-liquids. However, she had used nicotine salts during the previous week, which are formed by the reaction of nicotine with suitable acid, and are less volatile than freebase nicotine [7]. It is possible that the use of nicotine salts might have cause EVALI, although very recent reports suggest that vitamin E acetate may be the cause [8].

In summary, this first case of EVALI in Europe further supports the potential harmful effects of e-cigarettes, and should alert clinicians outside the USA about this treatable condition that will likely become more commonplace.

Gherzon Simón Casanova ${ }^{1}$, Rosanel Amaro ${ }^{1}$, Néstor Soler ${ }^{1,2,3}$, Marcelo Sánchez ${ }^{1,2}$, Joan Ramón Badía ${ }^{1,2,3}$, Joan Albert Barberà ${ }^{1,2,3}$ and Alvar Agusti ${ }^{1,2,3}$

${ }^{1}$ Hospital Clínic, Universitat de Barcelona, Barcelona, Spain. ${ }^{2}$ Institut d'Investigació Biomèdica August Pi I Sunyer (IDIBAPS), Barcelona, Spain. ${ }^{3}$ CIBER Enfermedades Respiratorias, Spain.

Correspondence: Alvar Agustí, Respiratory Institute, Hospital Clinic, Villarroel 170, 08036 Barcelona, Spain. E-mail: aagusti@clinic.cat

Received: 23 Oct 2019 | Accepted after revision: 23 Nov 2019

This article has editorial commentary: https://doi.org/10.1183/13993003.02419-2019

Conflict of interest: None declared.

\section{References}

1 Layden JE, Ghinai I, Pray I, et al. Pulmonary illness related to e-cigarette use in Illinois and Wisconsin preliminary report. N Engl J Med 2019; in press [https://doi.org/10.1056/NEJMoa1911614].

2 Carlos WG, Crotty Alexander LE, Gross JE, et al. Vaping-associated pulmonary illness (VAPI). Am J Respir Crit Care Med 2019; 200: P13-P14.

3 Henry TS, Kanne JP, Kligerman SJ. Imaging of vaping-associated lung disease. N Engl J Med 2019; 381: 1486-1487.

4 Maddock SD, Cirulis MM, Callahan SJ, et al. Pulmonary lipid-laden macrophages and vaping. N Engl J Med 2019; 381: 1488-1489.

5 McNeill A, Brose L, Calder R, et al. Evidence Review of E-cigarettes and Heated Tobacco Products. A Report Commissioned by Public Health England. London, Public Health England, 2018.

6 Butt YM, Smith ML, Tazelaar HD, et al. Pathology of vaping-associated lung injury. N Engl J Med 2019; 381: $1780-1781$.

7 Caldwell B, Sumner W, Crane J. A systematic review of nicotine by inhalation: is there a role for the inhaled route? Nicotine Tob Res 2012; 14: 1127-1139.

8 Blount B, Karwowski M, Morel-Espinosa M, et al. Evaluation of bronchoalveolar lavage fluid from patients in an outbreak of e-cigarette, or vaping, product use-associated lung injury - 10 states, August-October 2019. MMWR Morb Mortal Wkly Rep 2019; 68: 1040-1041. 